\title{
Dov M. GabBay \\ Semantics for Higher Level \\ Attacks in Extended \\ Argumentation Frames \\ Part 1: Overview
}

\begin{abstract}
In 2005 the author introduced networks which allow attacks on attacks of any level. So if $a \rightarrow b$ reads $a$ attacks $b$, then this attack can itself be attacked by another node $c$. This attack itself can attack another node $d$. This situation can be iterated to any level with attacks and nodes attacking other attacks and other nodes.

In this paper we provide semantics (of extensions) to such networks. We offer three different approaches to obtaining semantics.
\end{abstract}

1. The translation approach

This uses the methodology of 'Logic by translation'. We translate faithfully the new networks into ordinary Dung networks with more nodes and extract the semantics from the translation.

2. The labelling approach

This method regards the arrows as additional entities to be attacked and to mount attacks and applies a variation of the usual machinery of Camindada like labelling to the network. The new concept we need to employ here is that of 'joint attacks'.

3. The logic programming approach

We translate the higher level network into a logic program and obtain semantics for it through known semantics for logic programs.

We then compare our methods with those of S. Modgil and P. M. Dung et al.

Keywords: extended argumentations frames, semantics for attacks on attacks, joint attacks.

\section{Background}

In our 2005 paper [3] we introduced networks with higher level attacks of any kind. Figure 1 is a typical situation.

Figure 1 can represent any kind of network, not necessarily an argumentation network. It can be part of a Kripke model, an electrical network, a biological ecological network, etc. In each case the arrows have their own

Special Issue: New Ideas in Argumentation Theory

Edited by Dov M. Gabbay and Leendert van der Torre 


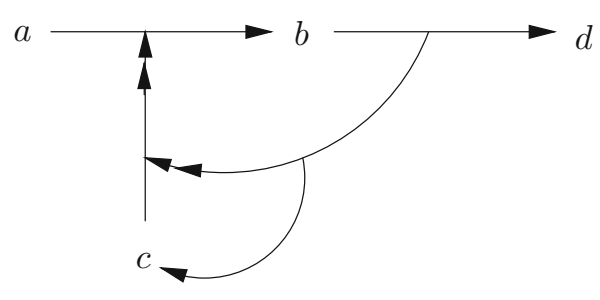

Figure 1.

meaning. The paper [3] also allowed for value annotations to the nodes and arrows and gave algorithms for the propagation of these values.

In the case of argumentation networks the nodes are arguments and the arrows mean attacks. The values (annotations) can correspond to the Caminada labellings and the propagation of values are governed by the properties of the Caminada labelling (see Definition 1.2 below for Caminada labellng).

The aim of this paper is to interpret (give semantics) to higher level attacks, in the case of argumentation networks.

Section 1 gives the background, Section 2 gives the conceptual ideas behind our approach, Section 3 gives the formal machinery of the model, Section 4 discusses other papers, Section 5 gives more formal results and Section 6 is the conclusion and discussion.

Let us look again at Figure 1, this time reading it as an argumentation frame.

In Figure 1 the argument $c$ attacks the attack from $a$ to $b$, (we use the notation $c \rightarrow(a \rightarrow b))$, while the attack from $b$ to $d$ attacks the attack emanating from $c$ (notation $(b \rightarrow d) \rightarrow(c \rightarrow(a \rightarrow b))$. This later attack attacks $c$ (notation $((b \rightarrow d) \rightarrow(c \rightarrow(a \rightarrow b))) \rightarrow c)$.

The question we ask is how to define the possible acceptable extensions for $\{a, b, c, d\}$ for the network of Figure 1 . The reader should note that whatever approach we give for defining exensions it must come from reasonable general principles which are meaningful for general networks. It should not rely on very specific features of argumentation networks. The general principles can have a specialised meaning in the argumentation case, but then equally the principles can have their own meanings in the case of other networks. We shall see later that we shall use general network fibring principles.

We now describe some recent background developments in the context of argumentation networks. Let us denote by $\mathbf{B} \mathbf{G} \mathbf{W}_{0}$ the argumentation networks where nodes are allowed to attack attacks. So let $S$ be a set of nodes. Denote by $x \rightarrow y, x, y \in S$, the attack of $x$ on $y$. Call this attack of 
level $(0,0)$. Assume $z$ is a node and $\alpha$ is an attack of level $(0, n)$ then $x \rightarrow \alpha$ is an attack of level $(0, n+1)$. The index 0 in $(0, n)$ indicates that attacks emanate from points $t \in S$.

Recently, in 2009, S. Modgil [15] used preferences to 'attack' or 'nullify' attacks from argument $a$ to argument $b$ on the grounds that $b$ is preferable to $a$.

Formally this gives rise to attacks on attacks of the form

$$
x \rightarrow(y \rightarrow z)
$$

Indeed, S. Modgil presented his system using our notation (arrows for attacks between arguments and double arrows for attacks on attacks) and presented ways of getting extensions for such networks.

His networks were required to satisfy additional conditions. This condition was motivated by the fact that the attacks on attacks come from preferences.

The Modgil condition is, (condition 2.4 in his paper [15]):

If $x$ attacks $a \rightarrow b$ and $y$ attacks $b \rightarrow a$ then $x$ attacks $y$ and $y$ attacks $x$.

$(x \rightarrow(a \rightarrow b))$ and $(y \rightarrow(b \rightarrow a))$ imply $(x \rightarrow y)$ and $(y \rightarrow x)$.

Let us denote the Modgil system by $\mathbf{M}$. The system $\mathbf{M}$ was addressed by D. D. Hahn, P. M. Dung and P. M. Thang in [14] who disagreed with the way S. Modgil was deriving his extensions, and presented an alternative way to derive extensions. Denote their system by HDT. At the same time, Dung called upon the authors of [3] to present a general semantics (for deriving extensions) for the general higher level case, especially their system BGW.

Quote from the end of Section 2 of [14]. ${ }^{1}$

Though the Modgil's extended argumentation could be viewed as a special case of BGW framework [3], its semantics is based on the underlining intuition that attacks against attacks represent preferences between conflicting arguments. Hence the condition 4 in Definition 2.4 is introduced. This constraint plays a fundamental role in the definition conflict-freeness and hence in Modgil's semantics. This insight suggests that different intuitions and applications could lead to different classes of extended argumentation and different semantics for general BGW extended argumentation.

\footnotetext{
${ }^{1}$ We changed the original bibliographical reference numbers to those of the present paper.
} 
Quote from Section 5 of [14]

We proposed a solution to an intriguing problem concerning the nonmonotonicity of the characteristic function of Modgil's acceptability. We believe that further study is needed to gain a better understanding of the semantics of extended argumentation frameworks, especially the general BGW extension of abstract argumentation. A key question in a semantics for BGW general extended argumentation is how the notion of conflict-free should be generalized and what does it mean for an argument to be acceptable? It would be interesting to see how works on logical modes of attacks [12] as well as interpretations in [3] could be applied to provide a formal framework here.

A similar comment was given in Section 6 of the paper of Baroni et al. [1]:

The idea of encompassing attacks to attacks in abstract argumentation framework has been first considered in [3], in the context of an extended framework encompassing argument strengths and their propagation. In this quite different context, deserving further development, Dung style semantics issues have not been considered.

Their approach is in fact what we suggest in Remark 2.7 of this paper. Call their approach BCGG. They were not aware of the work of Nielsen and Parsons [17] and so developed the machinery again.

Such higher level semantics is already implicitly available in our methodology, and this paper responds to Dung's call and presents our semantics explicitly.

Modgil and Dung disagree on the extensions of the networks of Figure 2 and Figure 3.

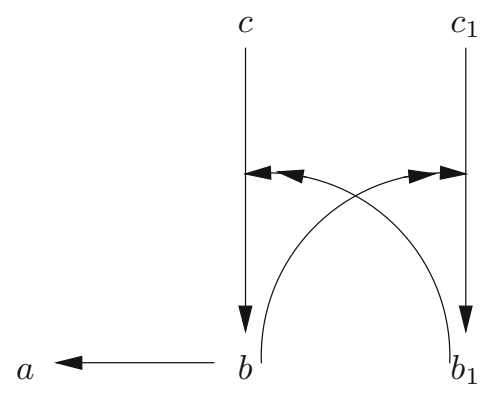

Figure 2 .

For the network of Figure 2, the acceptable $\mathbf{M}$ extensions are $\left\{c, c_{1}, a\right\}$ (which is not acceptable to HDT) and $\left\{c, c_{1}, b, b_{1}\right\}$. 


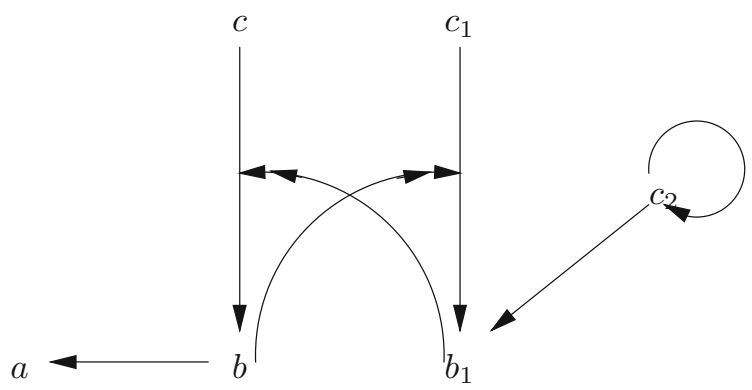

Figure 3.

For the network of Figure 3, $\mathbf{M}$ allows for the extension $\left\{c, c_{1}, a\right\}$ while HDT allows for the extension $\left\{c, c_{1}\right\}$. Our general approach will settle this disagreement from general principles.

To be able to present our case we need some definitions.

Definition 1.1 (Higher level argumentation frames).

1. An ordinary argumentation frame has the form $\mathbf{A}=(S, R)$, where $S$ is the set of arguments and $R \subseteq S \times S$ is the attack relation. ${ }^{2}$

2. A level $(0,1)$ argumentation frame has the form $\mathbf{A}^{0,1}=(S, \mathbb{R})$ where $S$ is the set of arguments and $\mathbb{R}$ is a set of pairs of the form $(x, y)$, or $(z,(x, y))$, where $x, y, z \in S .(x, y) \in \mathbb{R}$ means that $x$ attacks $y$ and $(z,(x, y)) \in \mathbb{R}$ means that $z$ attacks the attack $(x, y)$.

3. Level $(0, n)$ argumentation frames are defined as follows

(a) A pair $(x, y) \in S \times S$ is called a level $(0,0)$ attack.

(b) If $z \in S$ and $\alpha$ is level $(0, n)$ attack then $(z, \alpha)$ is a level $(0, n+1)$ attack.

(c) A level $(0, n)$ argumentation frame has the form $\mathbf{A}^{0, n}=(S, \mathbb{R})$ where $\mathbb{R}$ contains attacks $\beta$ of level $(1, m)$ for $m \leq n$, including some elements of level $(0, n)$.

Definition 1.2 (Camindada labelling). Let $\mathbf{A}=(S, R)$ be ordinary argumentation frame. A function $\lambda$

$$
\lambda: S \mapsto\{0,1, ?\}
$$

is called a complete Caminada labelling if it satisfies the following conditions:

\footnotetext{
${ }^{2}$ The usual notation is $\mathrm{Ar}=(\mathrm{Arg}, \mathrm{Att})$, but this notation becomes awkward when we move to higher levels.
} 
1. $\lambda(x)=1$ if for no $y$ do we have $y R x$

2. If for some $y, y R x$ and $\lambda(y)=1$. Then $\lambda(x)=0$

3. If for all $y$ such that $y R x$ we have $\lambda(y)=0$ then $\lambda(x)=1$.

4. If for all $y$ such that $y R x$ we have $\lambda(y) \neq 1$ and for some $y$ such that $y R x$ we have $\lambda(y)=$ ? then $\lambda(x)=$ ?.

5. If $\lambda(x)=1$ we say $x$ is 'live' or 'on'. If $\lambda(x)=0$, we say $x$ is 'dead' or 'out'. If $\lambda(x)=$ ? we say $x$ is 'undecided'.

REMARK 1.3 (Correspondence between Caminada labelling and extensions). There is a correspondence between Caminada labellings on $(S, R)$ and extensions on $S$. This is extensively studied in [7]. The following table 1 is taken from [7] and the references in it are for Definitions and Theorems in [7] itself.

\begin{tabular}{|c|c|c|}
\hline $\begin{array}{c}\text { restriction } \\
\text { complete labellings }\end{array}$ & $\begin{array}{c}\text { Dung-style } \\
\text { semantics }\end{array}$ & $\begin{array}{c}\text { linked by } \\
\text { def. and th. }\end{array}$ \\
\hline \hline no restrictions & complete semantics & Def. 5 and Th. 1 \\
\hline empty undec & stable semantics & Def. 8 and Th. 5 \\
\hline maximal in & preferred semantics & Def. 10 and Th. 7 \\
\hline maximal out & preferred semantics & Def. 10 and Th. 7 \\
\hline maximal undec & grounded semantics & Def. 9 and Th. 6 \\
\hline minimal in & grounded semantics & Def. 9 and Th. 6 \\
\hline minimal out & grounded semantics & Def. 9 and Th. 6 \\
\hline minimal undec & semi-stable semantics & Def. 11 and Th. 8 \\
\hline
\end{tabular}

Table 1. Argument labellings and Dung-style semantics

From now on we work with Caminada labellings. They are more convenient mathematically and more general conceptually. We can have labelling in general algebraic structures and not just into the set $\{1,0, ?\}$. This, however, is a story for another paper.

\section{Preliminary conceputal discussion}

We now discuss our options in giving semantics for attacks on attacks. We aim to analyse the basic situation of Figure 4 which is an acceptable network to $\mathbf{M}$.

However, we need to begin with a simpler figure first. 


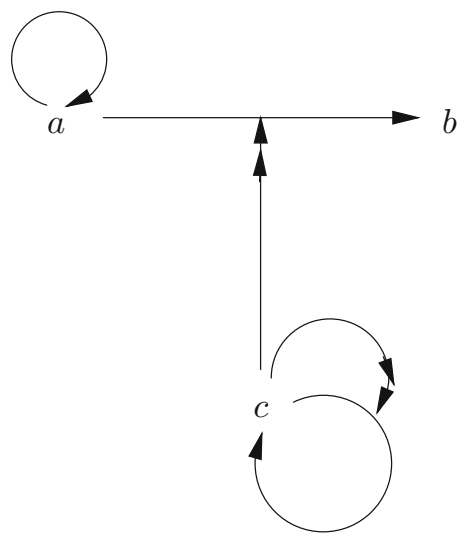

Figure 4.

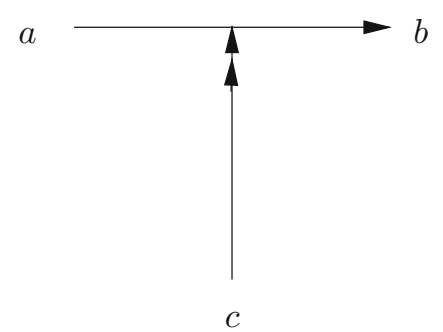

Figure 5.

\subsection{Conceptual discussion of attack on attack}

Consider Figure 5

We ask how many 'arguments' or 'units' are in this figure? We have $S=\{a, b, c\}$. Do we have more? We believe that ' $a \rightarrow b$ ' is a unit statement. It says that ' $a$ attacks $b$ '. The argument $c$ attacks this statement. It may be attacking it because $b$ is preferred to $a$, as in the S. Modgil approach $\mathbf{M}$, or it may be attacking it because $a$ is irrelevant to $b$. So our set of 'arguments' has not just the three elements $\{a, b, c\}$ but five elements $S^{*}=$ $\{a, b, c,(a, b),(c,(a, b))\}$. Each of the elements of $S^{*}$ can be 'live' or 'dead'.

To have a better appreciation of our problems let us follow a familiar story.

EXAmple 1 (The student and the exam). A girl student has one more exam to take for a degree. The course is Logic 101. She already has a job offer but she must get her degree to take up her position. She failed to show up to the exam and the university admin decided not to give her a degree. So her 
missing the exam turned up to be crucial to her getting a degree, since she was missing a mark in Logic for her total package of courses required for the degree. We have the following arguments at play:

$b=$ It is not possible to give a degree to the student because she is missing a grade in the Logic course.

The student attacks $b$ by giving two arguments

$a_{1}=\mathrm{I}$ was in hospital at the time and could not come to the exam.

$a_{2}=$ My grade average is good and even if I were to come to the exam and get grade 0 , I would still have had enough points for a degree.

The administration of the university countered the student's arguments as follows:

$c=$ The student was not in the hospital at the time of the exam. Hospital records do not show any admittance of her name.

$c$ obviously attacks $a_{1}$

$d=$ According to the rules of the university all students must take a Logic examination. It may be true that had she taken the exam and failed, she would have had enough points to get a degree, but her obligation was to take the exam and this she did not do.

Argument $d$ attacks not $a_{2}$ but the attack itself from $a_{1}$ onto $b$.

The student counters with two more arguments

$e=$ The university could not find a record of the student's admittance to the hospital because she was admitted to hospital under her married name, while at the univeristy she is registered under her maiden name.

Argument $e$ attacks argument $c$.

$f=$ you cannot apply the dry letter of university rules to cases where a person's entire future is in the balance. The application of this rule in this case is not fair and is therefore not valid.

Argument $f$ attacks the attack of $d$ on the attack of $a_{2}$ on $b$.

Figure 6 displays the situation in this case.

It is clear that this frame has one grounded extension

$$
\left\{d, f, a_{2}, e, a_{1}\right\}
$$

So the student will get her degree. 


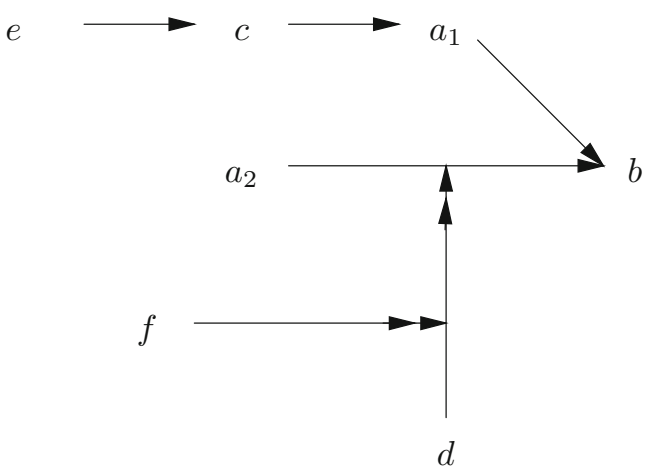

Figure 6.

From this example we see two points

1. Attacks on attacks need not necessarily be due to preferences.

2. The attack on attack can be valid even though the actual attack is not executed.

Put differently, $d$ for example, attacks the arrow from $a_{2}$ to $b$ in Figure 6 , and this arrow is independent of whether $a_{2}$ is 'live' or is being attacked and is 'dead'.

Consider Figure 7.

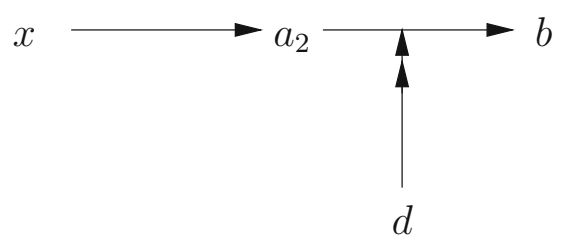

Figure 7.

In this figure, for the purpose of any extension involving $\left\{x, a_{2}, b, d\right\}$ the attack of $d$ on $a_{2} \rightarrow b$ is not relevant, because $a_{2}$ is 'dead', defeated by $x$. But if we allow ourselves to give the attack $a_{2} \rightarrow b$ an independent existence, $d$ plays a role in defeating this attack, independently of the staus of $a_{2}$.

To make the point even more clear and in focus, consider Figure 8.

In Figure 8, the arrow from $a_{2}$ to $b$ attacks some argument $y$. Clearly we want to know the acceptance of $a_{2} \rightarrow b$ as an attack independenlty of the accepance of $a_{2} \cdot{ }^{3}$

\footnotetext{
${ }^{3}$ Of course $\mathbf{M}$ and HDT are less concerned about this because they do not allow for
} 


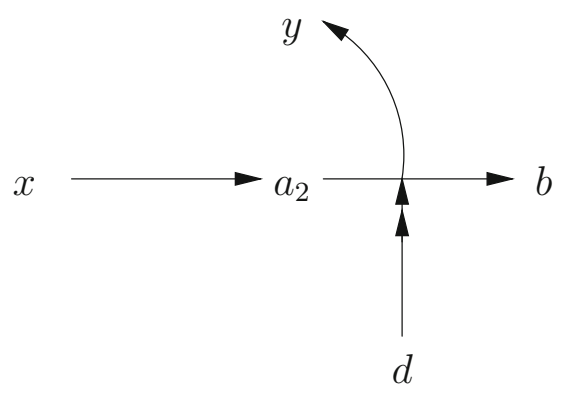

Figure 8.

Now consider Figure 4. What are our options for extensions?

We have one extension $\{c, a, b\}$, but we are not ready yet to explain why. We shall see later.

Let us now list the approaches we are going to take in providing semantics for higher level extended argumentation networks as defined in Definition 1.1. Let $(S, \mathbb{R})$ be a level $(0, n)$ argumentation network. We can provide semantics for it by one of the following methods.

\section{Translation option}

We faithfully embed the network $(S, \mathbb{R})$ of level $(0, n)$ into an ordinary argumentation frame $\left(S^{*}, R\right), R \subseteq S^{*} \times S^{*}$ such that $S \subseteq S^{*}$. The new ordinary network has more nodes. It satisfies certain conditions which enable us to extract semantics for $(S, \mathbb{R})$ from this embedding and from the traditional known semantics of $\left(S^{*}, R\right)$.

2. Labelling option

We extend the Caminada labelling concept to $(S, \mathbb{R})$, defining the notion of BGW labelling and using this BGW-labelling we define our exensions and give semantics for $(S, \mathbb{R})$. Alternatively one can consider an equivalent traditional Dung approach using the notions of conflict free sets, acceptability etc etc to get the extensions.

3. Logic programming option

We translate $(S, \mathbb{R})$ faithfully into a logic program $\pi$. The literals are the nodes $S \cup \mathbb{R}$. We use $\mathbb{R}$ to write the clauses of $\pi$. The semantics we get for $(S, \mathbb{R})$ comes from the semantics of logic programs.

attacks to emanate from arrows, however, we shall argue that there are cases of this nature in argumentation practice. Consider the case of Professor Smith who maintains he is not envious of the success of Professor Jones. Yet Smith attacks the theories of Jones at every opportunity. These repeated attacks may suggest that Smith is envious of Jones. 


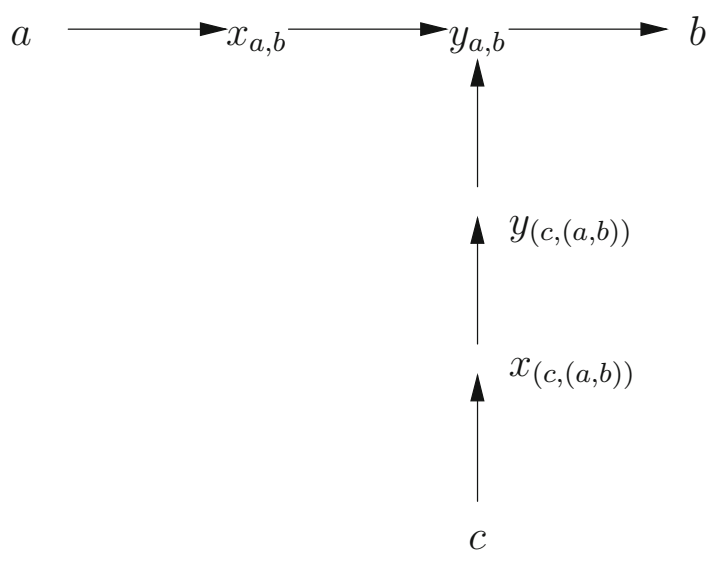

Figure 9.

\subsection{Discussion of the translation option}

We now give the reader a quick preview of our translation option, which will be discussed and motivated from general principles in the next section.

Consider Figure 5 again. Consider the expanded frame shown in Figure 9.

In Figure 9 we added for each attack arrow of the form $\alpha \rightarrow \beta$, two new points $x_{(\alpha, \beta)}$ and $y_{(\alpha, \beta)}$ and expanded the 'unit' $\alpha \rightarrow \beta$ into $\alpha \rightarrow x_{\alpha, \beta} \rightarrow$ $y_{\alpha, \beta} \rightarrow \beta$.

Note that if $\alpha$ is 'live' then $x_{\alpha, \beta}$ is 'dead' and so $y_{\alpha, \beta}$ is 'live' and so $\beta$ is 'dead'. The pair $\left(x_{\alpha, \beta}, y_{\alpha, \beta}\right)$ represents the attacking arrow from $\alpha$ to $\beta$. So to attack the arrow unit ' $\alpha \rightarrow \beta$ ' we attack $y_{\alpha, \beta}$. This is why the attacking $c$, which in Figure 5 attacks the arrow $a \rightarrow b$, in Figure 9 it attacks the point $y_{a, b}{ }^{4}$

In fact it is sufficient to do this trick only for arrows which are under attack. So for our purposes here (but not if we give a general mathematical definition), we can work with Figure 10, the simpler version of Figure 9.

We can similarly turn Figures 2 and 3 into Figure 11 and 12, resp.

Figures 11 and 12 are now ordinary frames. Let us check their extensions. We get the extensions for Figure 11.

$E_{1} \cdot\left\{c, c_{1}, y, y_{1}, a\right\}$

$E_{2} \cdot\left\{c, c_{1}\right\}$

$E_{3} .\left\{c, c_{1}, b, b_{1}\right\}$

\footnotetext{
${ }^{4}$ This trick is further exploited extensively in [6].

The scheme of Figure 10 was first independently presented by S. Modgil. See [16, 4].
} 


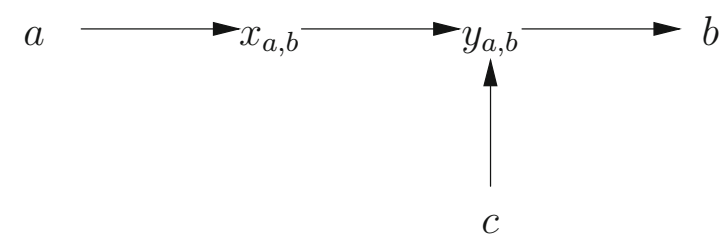

Figure 10.

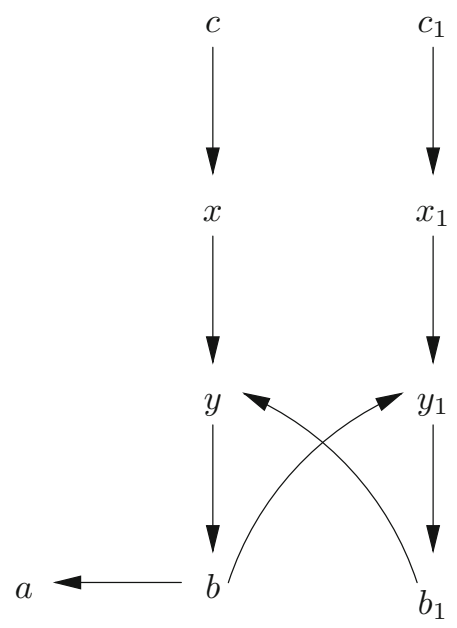

Figure 11.

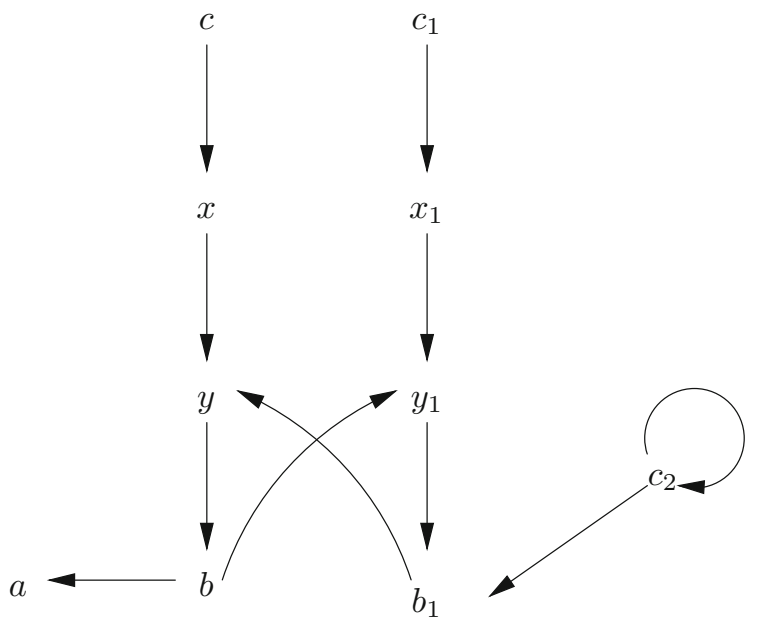

Figure 12. 
For the original language, $E_{1}$ suggests the extensio $\left\{c, c_{1}, a\right\}$.

For Figure 12 we get $E^{+}=\left\{c, c_{1}, y, y_{1}, a\right\}$.

$E^{+}$suggest the extension $\left\{c, c_{1}, a\right\}$ for the original language, i.e. we are supporting the Modgil semantics $\mathbf{M}$. We also have the extension $\left\{c, c_{1}\right\}$ supporting HDT.

Our strategy is therefore to start with an extended higher level frame based on the set $S$. We 'simplify' it by adding points $X$ of the form $x_{a, \alpha}, y_{a, \alpha}$ for any $(a, \alpha) \in \mathbb{R}$, we adjust the attacks as discussed and illustrated in Figures 9 and 10, and get an ordinary frame based on the points $S \cup X$.

Let $E_{1}^{+}, E_{2}^{+}, \ldots$ be the extensions on $S \cup X$. Then $E_{i}^{+} \cap S, i=1,2, \ldots$ are the extensions according to our translation option, on $S$.

The proper way to do this is in terms of Caminada labelling on $S \cup X$. The detailed machinery we develop in the next section.

The reader may now ask is this our solution? Are we happy now with the proposed translation option? The answer is no. We still need to continue with our conceptual analysis. We argued that we want to view ' $a \rightarrow b$ ' as an independent unit, the attack of $a$ on $b$, which can in itself be attacked by another argument $c$ or can itself attack another argument $d$. The 'trick' proposal of inserting the two intermediaries $x_{a, b}, y_{a, b}$ with $a \rightarrow x_{a, b} \rightarrow y_{a, b} \rightarrow$ $b$ may work for attacks from $c$ on $a \rightarrow b$, but it is not satisfactory for attacks from $a \rightarrow b$ onto other points $d$. Assume an attack from $a \rightarrow b$ onto $d$. Consider the trick translation of $a \rightarrow b$ into $a \rightarrow x_{a, b} \rightarrow y_{a, b} \rightarrow b$. We ask, where is this attack to emanate from? The obvious node is $y_{a, b}$. But if $a$ is not 'live', then $x_{a, b}$ is 'live' and $y_{a, b}$ is 'dead'. So it cannot attack other nodes. Our approach requires that ' $a \rightarrow b$ ' as a unit be kept 'live' unless attacked itself.

We therefore offer another solution. We adopt the view that in $a \rightarrow b$ the node $b$ is attacked by two arguments. The node $a$ and the attack arrow unit ' $a \rightarrow b$ '. This is a joint attack on $b$, both of these participants must be 'live'.

So how do we represent that? Figure 14 shows how it is done.

In Figure 14, ' $a \rightarrow b$ ' is just a node. We are saving on notation and not writing $z_{a, b}$. Consider now Figure 13. In Figure 13, only when both $a$ and ' $a \rightarrow b$ ' are 'live' will we have $y_{a, b}$ 'live' and hence $b$ is 'dead'. So Figure 13 is an implementation of the joint attack of ' $a$ ' and' $a \rightarrow b$ ' on ' $b$ ' of Figure 14 .

So to attack the arrow in $a \rightarrow b$ we attack ' $a \rightarrow b$ '. For the arrow of $a \rightarrow b$ to attack another point we emanate the attack from ' $a \rightarrow b$ '.

Let us now translate the frame of Figure 3 into our new set up, we get Figure 15. We need only use a simplified version. 


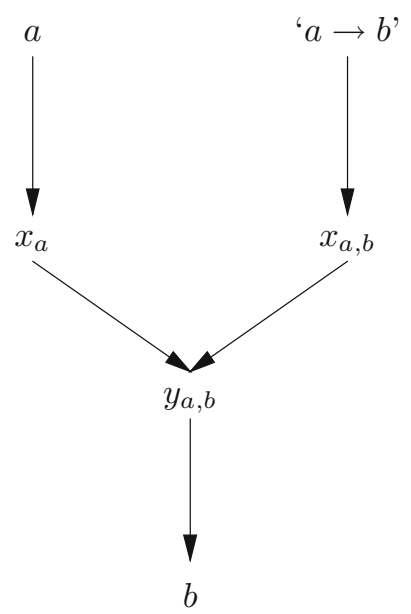

Figure 13. Representation of $a \rightarrow b$

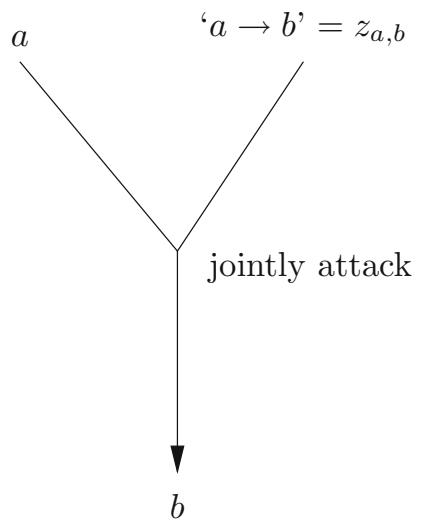

Figure 14. 


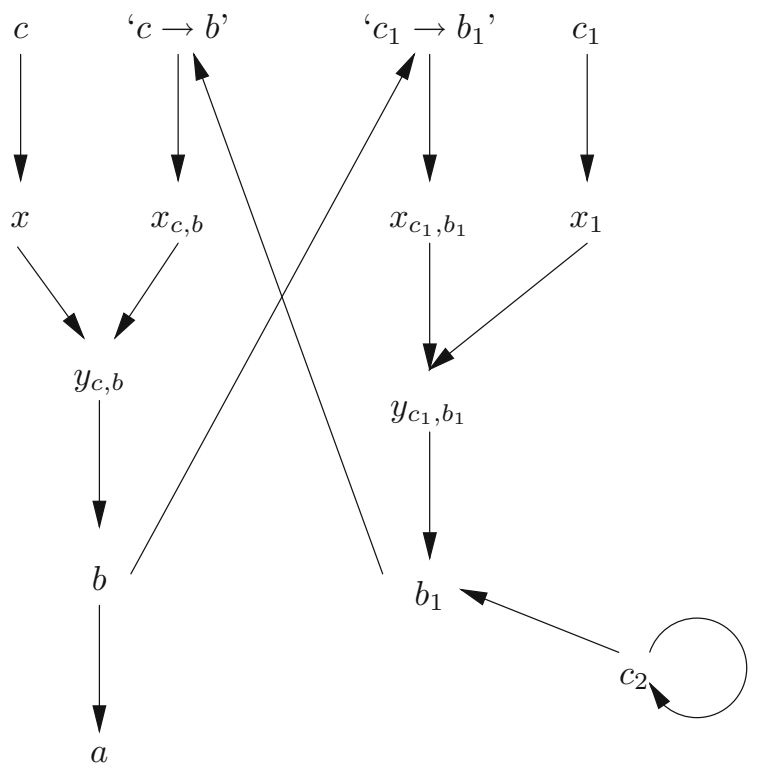

Figure 15.

Figure 15 is an ordinary argumentation frame. Let us calculate its extensions. In the original frame of Figure 3, we have the following extensions.

Modgil accepts $\left\{c, c_{1}, a\right\}$ only

Dung accepts $\left\{c, c_{1}\right\}$ only

1. Let us check Modgil option in Figure 15. If $a$ is 'live' then $b$ is 'dead', so ' $c_{1} \rightarrow b_{1}$ ' must be 'live'. Since $c_{1}$ is also 'live', then both $x_{1}$ and $x_{c_{1}, b_{1}}$ are 'dead' and so $y_{c_{1}, b_{1}}$ is 'live' and $b_{1}$ is 'dead'. So ' $c \rightarrow b$ ' is 'live'. So since $c$ is also 'live' we get that $x, x_{c, b}$ are 'dead'. Hence $y_{c, b}$ is live and $b$ is dead. The loop is complete and this is consistent.

2. Can we have an extension with only $\left\{c, c_{1}\right\}$ live? as Dung proposes for Figure 3? The answer is yes, we can.

REMARK 2.1 (Summary of our translation policy). Let $(S, \mathbb{R})$ be an extended higher level network. Transform it in some algorithmic way to an ordinary network $\left(S^{*}, R\right)$, such that the following holds

1. $S \subseteq S^{*}$

2. For every Caminada labelling $\lambda_{1}, \lambda_{2}$ on $S^{*}$ we have

$$
\lambda_{1} \uparrow S=\lambda_{2} \uparrow S \Rightarrow \lambda_{1}=\lambda_{2}
$$


(i.e. if $\lambda_{i}$ agree on all values on $S$ then they agree on $S^{*}$ as well. $)^{5}$

3. Then the extensions of $(S, \mathbb{R})$ are all subsets of the form $E_{\lambda}=\{x \in S \mid$ $\lambda(x)=1$ where $\lambda$ is a complete Caminada labelling on $\left.\left(S^{*}, R\right)\right\}$.

\subsection{The labelling option}

Consider the situation in Figure 5. We asked ourselves how many 'units' are participating in this network? The answer is five units.

$$
\{a, b, c, a \rightarrow b, c \rightarrow(a \rightarrow b)\}
$$

In the BGW labelling they all get labels in $\{0,1, ?\}$. So $a$ attacks $b$ through the channel ' $\rightarrow$ ' in ' $a \rightarrow b$ '.

For the attack to come through, $a$ must be 'live', and the channel must be 'live' and only then will $b$ be attacked.

In fact, annotating nodes and arrows is exactly what we were doing in [3] in 2005. We gave them real numbers and the numbers on the arrows were transmission and capacity indicators. See for example Figures 4 and 5 in [3]. We reproduce these figures here for the convenience of the reader.

Begin quotation from [3]:

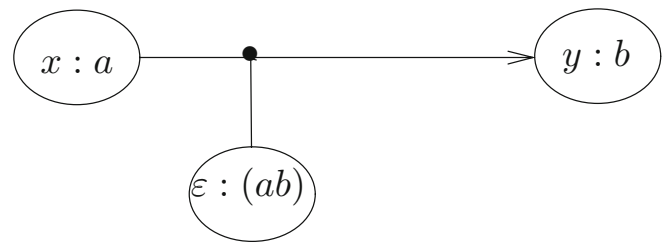

Figure 16. Fig 4 of [3]

$\varepsilon$ is the transmission factor, weakening $b$ in a way that takes account of $x: a$.

$b$ is also attacked by $d$ with factor $\beta$.

However, factor $\varepsilon$ is attacked by argument $c$, which is itself attacked by $d$, with transmission factor $\alpha$.

This model has two innovations.

1. The strength of nodes and the transmission factor.

2. The idea that the transmission factor can itself be attacked.

\footnotetext{
${ }^{5}$ This condition says that $S$ is a critical subframe of $S^{*}$. This notation was introduced in [9] and is studied extensively there. It ensures faithful embedding of the source in the target!
} 


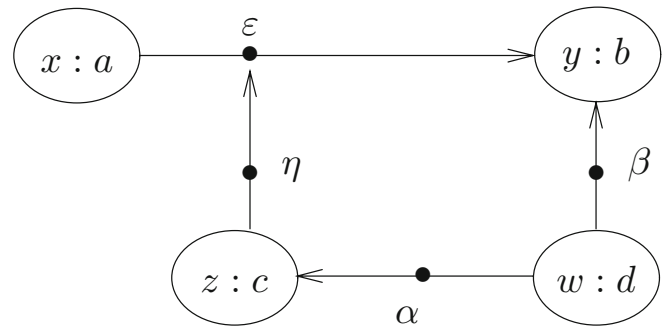

Figure 17. Fig 5 of [3]

What kind of network does Figure 17 represent? First, note that the strength of nodes is actually a colouring of them. One might expect us to introduce a transmission factor between colours, then in Figure $17 \varepsilon$ could depend only on $x$ and $y$. We choose to make $\varepsilon$ depend on the nodes, taking into consideration that the transmission factor depends on the nature of the argument and not just on their strengths.

The option of attacking transmission factors enables us to delete attacks, one by one, by attacking (lowering) their transmission factor.

End quotation from [3].

So we do the same here, the numbers are 'live' or 'dead', 'on', or 'off' indicators.

DeFinition 2.2 (BGW labelling). Let $(S, \mathbb{R})$ be a higher level argumentation frame. A function $\eta: S \cup \mathbb{R} \mapsto\{0,1, ?\}$ is a complete BGW labelling if the following holds.

We use Figure 18 for guidance. In Figure $18, a_{1}, \ldots, a_{k}$ are all the nodes attacking $\beta$ and for each arrow $a_{i} \rightarrow \beta$, the nodes $e_{1}, \ldots, e_{k_{i}}$ are all the nodes attacking it.

$\beta$ is either a node or an arrow of any level.

1. $\eta(\beta)=1$ if there is no $y$ such that $(y, \beta) \in \mathbb{R}$.

2. $\eta(\beta)=0$ if for some $a_{i}$ we have $\left(a_{i}, \beta\right) \in \mathbb{R}$ and $\eta\left(a_{i}\right)=1$ and $\eta\left(\left(a_{i}, \beta\right)\right)=$ 1 (i.e. both $a_{i}$ and the attack arrow are 'live').

3. $\eta(\beta)=1$ if for all $a_{i}$ such that $\left(a_{i}, \beta\right) \in \mathbb{R}$ we have that either $\eta\left(a_{i}\right)=0$ or $\eta\left(\left(a_{i}, \beta\right)\right)=0$.

4. $\eta(\beta)=$ ? if for all $a$ such that $(a, \beta) \in \mathbb{R}$ we have that either $[\eta(a)=0$ or $\eta(a, \beta)=0]$ or $[\eta(a)=1$ and $\eta(a, \beta)=$ ?] or $[\eta(a)=$ ? and $\eta(a, \beta)=1]$ or $[\eta(a)=\eta(a, \beta)=$ ?]. We also require that for some $a$ such that $(a, \beta) \in \mathbb{R}$ either $\eta(a)=$ ? or $\eta(a, \beta)=$ ? 


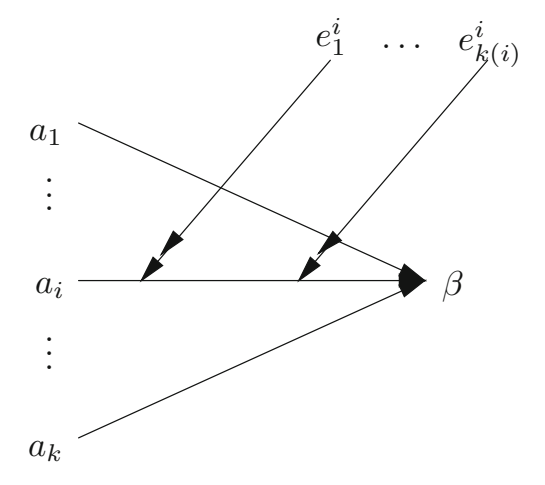

Figure 18.

We now stand at a crossroads of how to continue. Using the Caminada like BGW labelling we can define extensions as done in Table 1 and then use this to give semantics for $(S, \mathbb{R})$ or we can follow a more traditional route and talk about admissibility of sets $\mathbb{E}$, acceptability of elements relative to $\mathbb{E}$, extensions, etc., etc.

From the point of view of more generality and connections with other networks, the BGW labelling is better. From the point of view of simplicity in the particular cases of argumentation networks, the traditional way is better.

Luckily, there is a quick way to give semantics, using the connection we established in our discussion with joint attacks and the work already done by Nielsen and Parsons in [17].

Definition 2.3 (Frames with joint attacks). 1. A joint attack frame has the form $\mathbf{J}=(S, \mathcal{R})$ where $S$ is the set of arguments and $\mathcal{R} \subseteq S \times S \times S$ is a ternary relation. We understand $(x, y, z) \in \mathcal{R}$ as saying that the two nodes $(x, y)$ are mounting a joint attack on $z$. Note that the notation $\mathcal{R}$ allows us to make a distinction, say that $x$ is the main attacker and $y$ is the assistant attacker. However, for our purpose here we do not need this distinction.

2. We represent a joint attack diagrammatically as in Figure 19.

Figure 20 is an example of a network with joint attacks. Note that according to our definition, we can still have single attacks, if $(x, x, y) \in$ $\mathcal{R}$. So we generalise the standard networks.

The network of Figure 14 is another example. If we read the code in Figure 20 that $z_{\alpha, \beta}$ stands for ' $\alpha \rightarrow \beta$ ', then Figure 20 is a realisation of Figure 2 in terms of joint attacks. 


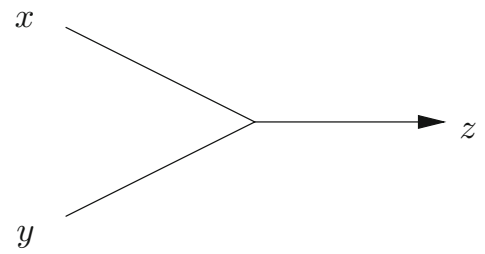

Figure 19.

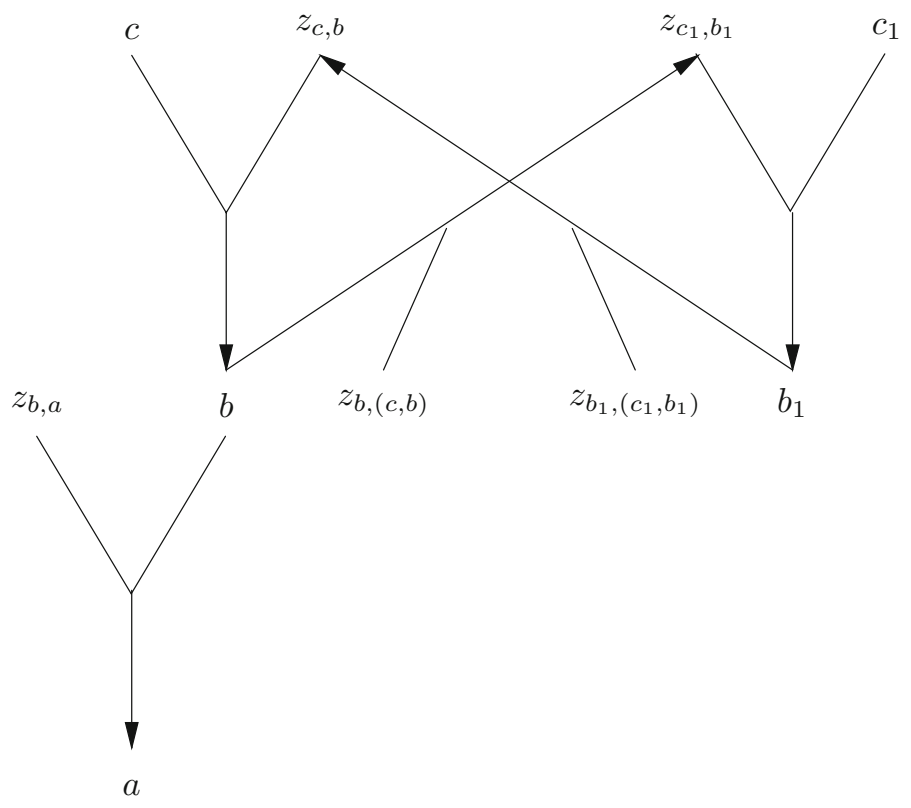

Figure 20.

DEFINITION 2.4 (Translation of an ordinary network into a joint attack network). Let $(S, R)$ be an ordinary network with $R \subseteq S \times S$. Define $\mathcal{R} \subseteq$ $S \times S \times S$ as follows

$$
\mathcal{R}=\{(x, y, y) \mid(x, y) \in R\} .
$$

DeFinition 2.5 (Reduction of higher order networks to joint attack networks). Let $(S, \mathbb{R})$ be a higher level network as defined in Definition 1.1. Define a corresponding joint argumenation frame $\left(S^{*}, \mathcal{R}\right)$ as follows:

$$
\begin{aligned}
& S^{*}=S \cup \mathbb{R} \\
& \mathcal{R}=\{(\alpha, \beta, \gamma) \mid \beta=(\alpha, \gamma) \text { and } \beta \in \mathbb{R}\} .
\end{aligned}
$$

If we look at Figures 2 and 20 , we see that we used $z_{\beta}$ to stand for $\beta$. 
It is now clear from the definitions that if we have the machinery for defining extensions for joint attack networks of Definition 2.3, then we can give semantics for higher level networks. Such machinery exists in [17], done beautifully and in detail by Nielsen and Parsons for general theory of sets of arguments attacking jointly other arguments. See their definitions and lemmas in pages 59-65. The next Definition 2.6 displays the necessary concepts for our case.

Definition 2.6. 1. A subset $\mathbb{E} \subseteq S \cup \mathbb{R}$ is conflict free if no $x \in \mathbb{E} \cap S$ and $(x, \beta) \in \mathbb{E}$ attacks any $\beta \in \mathbb{E}$.

2. $\beta$ is acceptable for $\mathbb{E}$ if for any $y$ such that $(y, \beta) \in \mathbb{R}$, there is a $z \in \mathbb{E}$ such that $(z, y) \in \mathbb{E}$ and $z \in \mathbb{E}$.

3. Let $\varphi(\mathbb{E})=\{\beta \mid \beta$ is acceptable by $\mathbb{E}\}$.

4. $\mathbb{E}$ is admissible if $\mathbb{E} \subseteq \varphi(\mathbb{E})$.

5. $\mathbb{E}$ is a preferred extension if $\mathbb{E}$ is maximal admissible.

6. $\mathbb{E}$ is a complete extension if $\mathbb{E}=\varphi(\mathbb{E})$.

7. $\mathbb{E}$ is stable if $\mathbb{E}$ attacks all $\beta \notin \mathbb{E}$.

Note that we reduced our problem of giving semantics to higher level networks of the form $(S, \mathbb{R})$ to the problem of giving semantics to the joint attack network $(S \cup \mathbb{R}, \mathcal{R})$ of Definition 2.5.

REMARK 2.7 (Answering Dung's question). We can now give a very short answer to Dung's question from [14].

HDT say (I quote):

"A key question in a semantics for BGW general extended argumentation is how the notion of conflict-free should be generalised and what does it mean for an argument to be acceptable"

My quick answer: Start with higher level $(S, \mathbb{R})$ of Definition 1.1. Translate into a joint attack network of Definition 2.3, using the translation of Definition 2.4. Apply the machinery of [8] as suggested in Definition 2.6 and now you are done.

REMARK 2.8. We comment in passing that networks with joint attacks have been studied in [9]. We have in [9] joint and disjunctive attacks as in Figure 21. Disjuntive attack of $y$ on $e_{1}, \ldots, e_{m}$ requires that if $y$ in live then at least one of $e_{i}$ is dead. This is not the same as the disjunction of the separate attacks of $y$ on each $e_{i}$. 


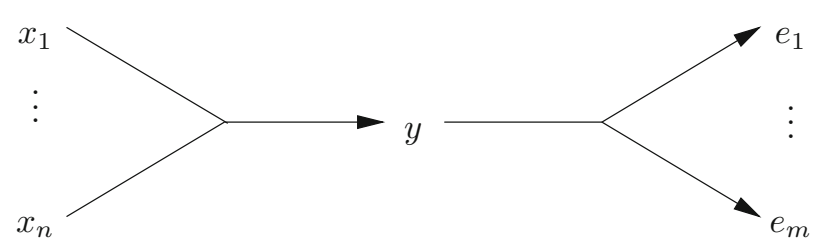

Figure 21.

REMARK 2.9 (Comparison with [1]). We compare with the approach of BCGG. They, like ourselves, regard attacks as objects and put them into the argumentation network. Thus if $E$ is a set of arguments and attacks and $x$ is a node attacked by $y$, then $E$ defends $x$ according to BCGG if $E$ attacks either $y$ or the attack from $y$ to $x$. Since $x$ is being attacked jointly by $y$ and by ' $y \rightarrow x$ ', the kind of defence BCGG are offering means that according to them to attack a joint set $\{u, v\}$ we need to attack one of the members, either $u$ or $v$. This is the same notion as in [17]. We have already argued in [9] that to attack a joint set $\{u, v\}$ we need the concept of disjunctive attack. In our system we can allow for $E$ to mount a disjunctive attack on the set $D=\{y$, attack of $y$ on $x\}$. Disjunctive attacks are different. See Figure 21, and Remark 2.8. We can also attack the joint attack of $D$ as shown in Figure 22. Our methodology requires we chose the approach which works for a variety of networks - not just for argumentation frames. There is a distinction here to be made within our methodology, which does not arise in the narrow argumentation context. When $x$ attacks $y$ and we want to attack this attack of $x$ on $y$, what are we attacking? Are we attacking the argument of the attack from $x$ to $y$ ? E.g. saying $y$ is preferable to $x$ ? Or are we attacking the transmission channel which transmits the attack as in Figure 22? The latter makes sense in other networks, mentioned in [3]. ${ }^{6}$

REMARK 2.10 (Higher level joint attack networks). Of course we can allow attacks on joint attacks as well, as in Figure 22.

This can be iterated and can get quite involved, but this is the subject of another paper.

REMARK 2.11 (Discussion of the Modgil-Dung approach). Consider Figure 23.

\footnotetext{
${ }^{6}$ Imagine a prosecutor bringing in new evidence $a$ and arguing its relevance to the case and claiming (' $a \rightarrow b$ ') that it shows that the defence claims $b$ is false. The defence can attack the actual evidence (attack $a$ ) or question the relevance of the evidence to $b$ (attack ' $a \rightarrow b$ '). The defence can also claim the new evidence cannot be introduced at this stage of the proceedings. This is an attack on the channel.
} 


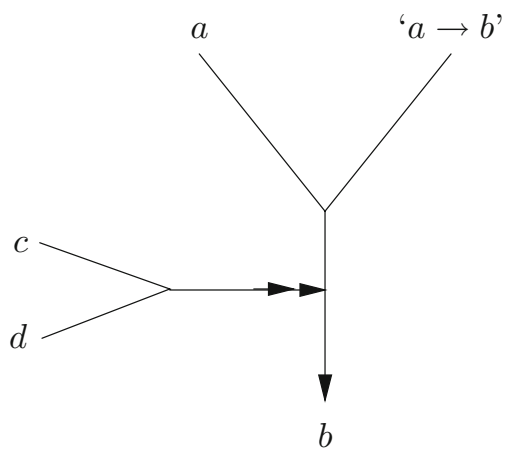

Figure 22 .

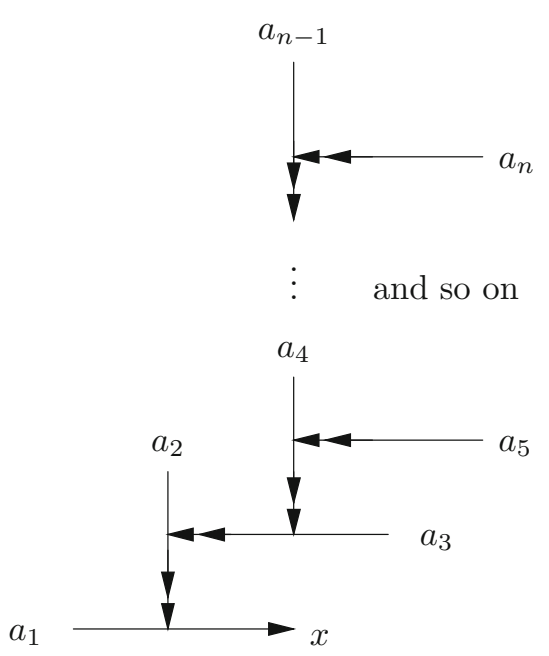

Figure 23.

The attack arrows of the form

$$
\begin{aligned}
& \beta_{1}=a_{1} \rightarrow x \\
& \beta_{2}=a_{2} \rightarrow \beta_{1} \\
& \beta_{n}=a_{n} \rightarrow \beta_{n-1}
\end{aligned}
$$

are considered in our labelling approach as independent entities (also by Baroni et al. [17]) and thus have to be treated uniformly as elements of the network $S \cup \mathbb{R}$ (see Definition 2.6). $\beta_{1}, \ldots, \beta_{n}$ loose their individuality in this approach. Not so in the translation approach and in the Dung-Modgil approaches. In the tranlsation approach (see Remark 2.1), S is part of $S^{*}$ and so all nodes appear as they are. 
In the Dung approach the notion of defence is inductive. So we have to say explicitly and inductively that

$$
\begin{aligned}
& \beta_{2} \text { defends } x \\
& \beta_{3} \text { defends } \beta_{1} \\
& \beta_{4} \text { defends } \beta_{2}
\end{aligned}
$$

and so on.

Whether $x$ is eventually defended or not by the $\beta_{i}$ sequence depends on how the sequence ends.

So a set $E$ defends $x$ if $E$ contains the correct inductive sequence. This approach, like the translation approach, maintains the individuality of the attacks and allows us, if we so wish, to have all kinds of different attacks in the sequence. So, for example, $a_{2}$ can be an entire new network coming to defend $x$.

Thus the Dung approach is different from that of Baroni et al. [17] and from our labelling approach. It can be generalised in different ways and I would like to consider it as a fourth approach in the list of approaches we gave at the end of Section 2.1.

\subsection{The logic programming approach}

Let $(S, \mathbb{R})$ be a higher level extended program and let $S^{*}=S \cup \mathbb{R}$. We write a translation logic program $\pi$ for $(S, \mathbb{R})$ as follows.

1. The literals of $\pi$ are all the elements of $S^{*}$.

2. For each $\beta \in S^{*}$, if $\beta$ is not attacked then take the clause $\beta$.

3. If $\beta$ is attacked then Figure 18 is the typical situation.

Take the clause

$$
\left.\beta \text { if } \bigwedge_{i=1}^{k}\left(\neg a_{i} \vee \bigvee_{j=1}^{k(i)}\left(e_{j}^{i},\left(a_{i}, \beta\right)\right) \wedge e_{j}^{i}\right)\right)
$$

We have

(a) $s \in S$ is in the ground extension iff $\pi \vdash s$

(b) $\mathbb{E}$ is an extension of $S^{*}$ iff $\mathbb{E}$ is an answer set solution in $\pi$.

The sets $\mathbb{E} \cap S$ gives for all extensions $\mathbb{E}$ give the extensions for $S$.

We now conclude this overview. In part 2 of this paper [11] we shall develop the following: 
- We shall develop the mathematics underlying the three approaches (translation approach, labelling and extensions fixed points approach and the logic programming approach).

- We shall compare the three approaches and hope to show that all three approaches are equivalent.

We also show that we favour the Dung approach for the case of level $(0, n)$ (Dung's level).

- We shall provide semantics for higher level networks where arrows can attack other arrows.

- We shall place our results within the landscape of our general network methodology.

Acknowledgements. I am grateful to P. M. Dung for reading and commenting on the paper. In particular Dung disagrees with my analysis of Example 1 of page 361. Dung believes that by itself, the reasoning represented as $b$ is not an argument. It is an argument only because of the regulation expressed in $d$, saying that the university rules state that taking logic is an obligation for graduation. Therefore Dung would rather say that $(b, d)$ together is an argument. $d$ contains two parts, one of them is the regulation that allows $b$ to become an argument and the other is just a statement saying that $a_{2}$ is an argument. Furthermore, while $a_{2}$ attacks $b$, $b$ also attacks $a_{2}$. In other words, $a_{2}$ and $b$ attack each other. Dung also claims that $f$ is an attack against $b$.

\section{References}

[1] Baroni, P., F. Cerutti, M. Giacomin, and G. Guida, 'Encompassing attacks to attacks in abstract argumentation frameworks', ECSQARU 2009, LNAI 5590, Springer, 2009, pp. 83-94.

[2] Barringer, H., D. M. Gabbay, and S. Modgil, 'Modal and Temporal Argumentation Networks'. To appear.

[3] Barringer, H., D. M. Gabbay, and J. Woods, 'Temporal Dynamics of Argumentation Networks', in D. Hutter and W. Stephan (eds.), Volume Dedicated to Joerg Siekmann, Mechanising Mathematical Reasoning, Springer Lecture Notes in Computer Science 2605, 2005, pp. 59-98.

[4] Bench-Capon, T., and S. Modgil, 'Case Law in Extended Argumentation Frameworks', in ICAIL'09: Proceedings of the 12th International Conference on Artificial Intelligence and Law, Barcelona, Spain, ACM, New York, 2009, pp. 118-127. IJAIL 08.

[5] Boella, G., D. M. Gabbay, L. van der Torre and S. Villata, 'Meta-Argumentation, part 1.', Studia Logica, 93(2-3): 297-355, 2009, this issue. 
[6] Boella, G., D. M. Gabbay, L. van der Torre and S. Villata, 'Argumentation Networks with Voluntary Attacks', in preparation.

[7] Caminada, M., and D. M. Gabbay, 'A logical account of formal argumentation', Studia Logica, 93(2-3): 109-145, 2009, this issue.

[8] Caminada, M., and D. M. GabBay, 'Embedding logic programs in argumentation framework', Studia Logica, 93(2-3): 383-403, 2009, this issue.

[9] Gabbay D. M., 'Fibring Argumentation Frames', Studia Logica, 93(2-3): 231-295, 2009, this issue.

[10] Gabbay, D. M., 'Modal Provability Foundations for Argumentation Networks', Studia Logica, 93(2-3): 181-198, 2009, this issue.

[11] Gabbay D. M., 'Semantics for higher level attacks in extended argumentation frames', part 2.

[12] Gabbay D. M., and A. S. D'Avila Garcez, 'Logical modes of attack in argumentation networks', Studia Logica, 93(2-3): 199-230, 2009, this issue.

[13] Gabbay D. M., and A. Szazas, 'Annotation theories over graphs', Studia Logica, 93(2-3): 147-180, 2009, this issue.

[14] Hanh, Do Duc, Phan Minh Dung, and Phan Minh Thang, 'Inductive Defense for Modgil's Extended Argumentation Framework'. Submitted to Journal of Logic and Computation.

[15] Modgil, S., 'Reasoning about preferences in argumentation frameworks', Artificial Intelligence, 173(9-10): 901-934, 2009.

[16] Modgil, S., and T. Bench-CAPon, 'Integrating Object and Meta-level Value Based Argumentation', Proceedings of the 2nd International Conference on Computational Models of Argument, 2008, pp. 240-251. Expanded version available at: www.csc. liv.ac.uk/research/techreports/tr2009/tr09019abs.html

[17] Nielsen, S. H., and S. PARsons, a Generalization of Dung's Abstract Framework for Argumentation: Arguing with Sets of Attacking Arguments, LNCS Vol 4766, Springer, 2007, pp. 54-73.

Dov M. GabBay

Department of Computer Science

King's College, London, UK

Bar-Ilan University, Israel

University of Luxembourg

dov.gabbay@kcl.ac.uk 